Voix et Images

\title{
Bibliographie critique des études consacrées aux romans d'Anne Hébert
}

\section{Janet M. Paterson}

Volume 5, numéro 1, automne 1979

Jacques Godbout

URI : https://id.erudit.org/iderudit/200196ar

DOI : https://doi.org/10.7202/200196ar

Aller au sommaire du numéro

Éditeur(s)

Les Presses de l'Université du Québec

\section{ISSN}

0318-9201 (imprimé)

1705-933X (numérique)

Découvrir la revue

\section{Citer ce document}

Paterson, J. M. (1979). Bibliographie critique des études consacrées aux romans d'Anne Hébert. Voix et Images, 5(1), 187-192. https://doi.org/10.7202/200196ar d'utilisation que vous pouvez consulter en ligne.

https://apropos.erudit.org/fr/usagers/politique-dutilisation/ 


\section{Bibliographie critique des études consacrées aux romans d'Anne Hébert}

Cette bibliographie recense les principales études consacrées aux romans d'Anne Hébert, surtout les livres, les articles et les thèses. Sont omis les articles de presse, les comptes rendus et les allusions dans les histoires de la littérature.

\section{Romans d'Anne Hébert}

Les Chambres de bois, Paris, Seuil, 1958.

Kamouraska, Paris, Seuil, 1970.

Les Enfants du sabbat, Paris Seuil, 1975.

\section{Etudes sur les romans}

ALLARD, Jacques "Les Enfants du sabbat d'Anne Hébert", Voix et images, I no 2 (décembre 1975) pp. 285-286. Aperçu favorable du roman qui souligne certaines données formelles, à savoir, le découpage en lexies et le jeu des voix diverses.

AMAR, Wenny, “L'Amour dans l'œuvre d'Anne Hébert", thèse de maîtrise, Université McGill, 1975. Étude descriptive des différentes formes de l'amour dans l'œuvre hébertienne. Travail intéressant surtout pour le rapport qu'il démontre entre l'amour et la mort.

AYLWIN, Ulric, "Vers une lecture de l'œurre d'Anne Hébert", La Barre du jour, no 7 (été 1966) pp. 2-11. Introduction à une étude stylistique et sémantique des Chambres de bois; tient compte des articles, des conjonctions, des locutions, des métaphores, des temps verbaux, des propositions et des phrases.

AYLWIN, Ulric, "Au pays de la fille maigre: Les Chambres de bois d'Anne Hébert", Voix et images du pays I, Les Cahiers de l'Université du Québec, 1970, pp. 37-50. Cet article examine les espaces extérieurs et intérieurs du roman et les relie aux ténèbres d'une société.

BISHOP, Neil Breton, "La Thématique de l'enfance dans l'œuvre poétique et romanesque d'Anne Hébert", thèse de doctorat, Université d'Aix-en-Provence, 1977. Une approche thématique qui cerne systematiquement les caractéristiques majeures du monde de l'enfant et qui examine rigoureusement l'évolution du personnage hébertien en fonction d'une problématique de la sortie de l'enfance. 
BLAIN, Maurice, "Anne Hébert ou le risque de vivre", Liberté '59, no 5 (septembreoctobre 1959) pp. 322-330, et dans Gilles Marcotte, Présence de la critique, Montréal, H.M.H., 1966, pp. 155-163. L'une des premiers essais à étudier certains aspects formels des Chambres de bois. Analyse aussi les thèmes de la mort et de la libération en fonction de l'œuvre entière.

BODART, Marie-Thérèse, "Anne Hébert, canadienne-française", Synthèses, Paris, Bruxelles, nos 292-293 (octobre-novembre 1970) pp. 121-125. Non vidi.

BORDAZ, Robert, "Anne Hébert et le roman canadien ", La Revue des deux mondes, no 6,1971 , pp. 617-622. Critique élogieuse du roman Kamouraska perçu comme caractéristique de la nouvelle littérature canadienne. Résumé de l'intrigue et quelques précisions sur le style.

BOUCHARD, Denis, “Érotisme et érotologie, aspects de l'œuvre poétique et romanesque d'Anne Hébert", Revue du Pacifique, I no2 (automne 1975) pp. 152-167. Repris avec des variantes dans Une lecture d'Anne Hébert, Cahiers du Québec/Hurtubise HMH, 1977, pp. 133-148.

“Les Enfants du sabbat d'Anne Hébert: l'enveloppe des mythes», Voix et images, 1 no 3 (avril 1976) pp. 374-385. Repris avec des variantes dans Une lecture d'Anne Hébert, Cahiers du Québec/Hurtubise HMH, 1977, pp. 177-191.

"Anne Hébert et la "Solitude rompue" "Etudes françaises, 13 nos 1-2 (avril 1977) pp. 163-179. Repris avec des variantes dans Une lecture d'Anne Hébert, Cahiers du Québec/Hurtubise HMH, 1977, pp. 55-70.

"Anne Hébet et le "Mystère de la parole", un essai d'antibiographie”, Revue du Pacifique, III no 1 (printemps 1977) pp. 67-81. Repris avec des variantes dans Une lecture d'Anne Hébert, Cahiers du Québec/Hurtubise HMH, 1977, pp. 13-29.

Une lecture d'Anne Hébert, Cahiers du Québec/Hurtubise HMH, 1977. Le sous-titre "La Recherche d'une mythologie" annonce un des deux aspects principaux de cette étude: la mise en rapport des mythes dans l'œuvre hébertienne avec ceux de la collectivité québécoise. Au dire de l'auteur, cette œuvre représente "une analyse profondément subtile de notre collectivité " (p. 5). A cette étude s'ajoute une interprétation d'ordre génétique selon laquelle la personne et l'œuvre de Saint-Denys Garneau auraient profondément marqué l'écriture d'Anne Hébert. Le livre se divise en deux parties: la première partie examine la poésie par rapport au poème "Le Tombeau des rois" et la deuxième partie étudie les romans à la lumière des Enfants du sabbat. L'analyse de la prose porte surtout sur l'érotisme, la sublimation et la cruauté, éléments qui relèveraient d'une dualité inhérente à la structure fondamentale de l'œuvre. L'auteur souligne aussi les cótés comiques et allégoriques des Enfants du sabbat. A la fin se trouve une longue bibliographie consacree aux articles de presse des pays francophones. Pour une critique de ce livre voir: Henri-Paul Jacques, "A propos d'Une lecture d'Anne Hébert", Voix et images, III no 3 (avril 1978) pp. 448-458.

BUCKNALL, Barbara J., "Anne Hébert et Violette Leduc, lectrices de Proust ", Bulletin de I'A.P.F.U.C. (Association des professeurs de français des universités canadiennes), février 1975, pp. 83-100. Étude peu convaincante de l'influence proustienne sur l'esthétique d'Anne Hébert. Au sujet des souvenirs et de l'amour passion, l'auteur établit des rapports douteux entre Les Chambres de bois, Kamouraska et $A$ la recherche du temps perdu.

CHAMPAGNE, Jean-Marc, "L'Enfance dans les romans d'Anne Hébert", thèse de maîtrise, Université du Québec à Trois-Rivières, 1973. Étude descriptive du 
thème de l'enfance. Débouche sur des interprétations biographiques et sociologiques très impressionnistes.

CHIASSON, Arthur Paul, «The Tragic Mood in the Words of Anne Hébert ", thèse de doctorat, Tufts University, Massachusetts, 1974. Discussion de l'élément tragique dans l'cuure hébertienne. Prend comme point de départ l'image du faucon aveugle dans "Le Tombeau des rois". Introduction historique, analyse de l'oeuvre, conclusion décevante qui se rapporte à l'aliénation de la société canadienne-française et à la littérature du tiers monde.

COHEN, Henry, "Le Rôle du mythe dans Kamouraska d'Anne Hébert", Présence francophone, no 12 (printemps 1976) pp. 103-111. Essai original et intelligent qui examine le jeu des mythologies dans Kamouraska en se rapportant aux mythes du Canada français, aux contes de fées et à l'antiquité grecque.

COLENO, Gilbert, «Le Milieu physique dans l'œuvre d'Anne Hébert», diplôme d'études supérieures, Université de Montréal, 1971. S'intéresse aux rapports entre les personnages et le monde physique tout en soulignant l'omniprésence de l'aliénation dans l'ceuvre. Propose une conclusion peu convaincante qui porte sur la libération personnelle de l'auteur.

DORAIS, Fernand, "Kamouraska d'Anne Hébert - essai de critique herméneutique». La Revue de l'Université laurentienne, IV no 1 (novembre 1971) pp. 76-82. Article très rapide qui se propose "d'expliciter les conditions d'existence du roman» mais qui ne fournit que quelques commentaires d'ordre impressionniste.

DUFRESNE, Françoise M., "Le Drame de Kamouraska», Québec-Histoire, nos 5-6 (maijuin-juillet 1972) pp. 72-77. Commentaire critique et citations de journaux de l'époque au sujet du fait réel à la source du roman, à savoir, l'assassinat d'un dit Achille Taché le 31 janvier 1839. Cet article signale la présence d'un premier roman sur ce sujet, notamment Drames de la vie réelle de Georges-Isidore Barthes, 1896. Source documentaire capitale.

FERAL, Josette, "Clôture du moi, clôture du texte dans l'ceuvre d'Anne Hébert", Voix et images, I no 2 (décembre 1975) pp. 265-283, et dans Littérature, no 20 (décembre 1975) pp. 102-117. Essai dense et original et l'un des seuls à se rattacher à la dimension littérale des textes. Analyse dans Kamouraska la superposition de deux ordres chronologiques du discours, étudie la clôture du texte au niveau de la narration et examine l'imbrication des temps.

FULTON, Barbara, "Kamouraska: le vide au centre", thèse de maîtrise, University of British Columbia, 1976. Aborde l'analyse de Kamouraska d'un point de vue structural dans le but de montrer que toutes les structures se résument dans la révélation du vide par l'antithèse. Étudie dans cette perspective les thèmes du masque, du circulaire et de la culpabilité. Malheureusement, cette thèse possède de grandes lacunes sur le plan méthodologique qui empêchent une analyse rigoureuse du sujet.

GODIN, Jean-Cléo, "Rebirth in the World", Yale French Studies, no 45 (novembre 1970) pp. 137-153. Étude générale de l'ceuvre qui se concentre surtout sur les poèmes. Signale les motifs du rêve, de l'absence et de l'aliénation. Brève discussion des Chambres de bois.

GOSSELIN, Michel, "Étude du discours narratif dans Kamouraska d'Anne Hébert", thèse de maîtrise, Université de Sherbrooke, 1974. A l'appui d'une analyse formelle, cette thèse cherche à dégager l'essentiel de la technique narrative dans Kamouraska. Examine systématiquement la voix et le mode mais sans pour cela toujours tirer des conclusions justes ou pertinentes. 
JUERY, René Y.F., «Oeuvres en prose d'Anne Hébert - Essai de sémiotique narrative et discursive de "La Robe corail" et de Kamouraska", thèse de doctorat, Université d'Ottawa, 1977. S'inspirant des travaux formalistes et plus particulièrement de ceux de Greimas, cette thèse applique une analyse sémiotique à ces deux textes. Grâce à une description exhaustive, l'auteur rend compte des articulations des contenus. Ce travail, rigoureux et systématique, cherche à éprouver une méthodologie plutôt qu'à trouver des sens nouveaux dans la prose hébertienne.

LACOTE, René, Anne Hébert, Paris, Editions Seghers, 1969. Ouvrage d'initiation à l'œuvre: vie, étude, choix de poèmes, illustrations et chronique bibliographique. Au sujet des Chambres de bois, ce critique signale les dimensions poétiques et oniriques du roman.

LEBLOND, Sylvio, “Le Drame de Kamouraska d'après les documents de l'époque ", Les Cahiers des dix, no 37 (1972) pp. 239-273. Cet article, comme celui de Françoise Dufresne qui le précède, met en évidence les éléments du fait réel du drame de Kamouraska qui a eu lieu en 1839. Décrit aussi les personnages selon certains documents de l'époque.

LEFEVRE, Louisette, "Le Thème de la femme dans l'œuvre romanesque d'Anne Hébert ", thèse de maîtrise, Université du Québec à Trois-Rivières, 1976. L'auteur adopte un point de vue symbolique pour examiner les personnages féminins. Etudie avec rigueur les différentes étapes dans la vie de ces personnages, analyse avec finesse leurs grands complexes, à savoir, l'angoisse, la culpabilité et la solitude et met en lumière les manifestations de la révolte féminine par l'amour, la haine et la mort.

LE GRAND, Albert, “Anne Hébert : de l'exil au royaume», dans Littérature canadiennefrançaise (Conférences J.-A. de Sève), Montréal, Presses de l'Université de Montréal, 1969, pp. 181-213 et dans Études françaises, IV no 1 (février 1968) pp. 3-29. Une très bonne étude dont l'orientation est socio-critique. Perçoit l'œuvre comme un univers dont les signes représentent ceux d'un destin personnel et collectif.

"Une Parole enfin libérée», Maintenant, nos 68-69 (septembre 1967) pp. 267-272. Article général portant sur la révolte des écrivains dans la littérature québécoise.

, Kamouraska ou l'ange et la bête", Études françaises, VII no 2 (mai 1971) pp. 19-143. Analyse très subtile du roman à partir du motif du fait divers. Celui-ci déclencherait toute la fiction aux niveaux du dédoublement du personnage principal, des temps et de la triple articulation de la mémoire, du songe et du sommeil.

LE MOYNE, Jean, "Hors les Chambres d'enfance", dans Gilles Marcotte, Présence de la critique, Ottawa, H.M.H., 1966, pp. 35-43. Étude socio-critique qui met la libération dans le roman en rapport avec celle de la conscience littéraire québécoise.

MARMIER, Jean, "Du Tombeau des rois à Kamouraska: vouloir vivre et instinct de mort chez Anne Hébert". Missions et démarches de la critique, librairie C. Klincksieck, 1973, pp. 807-815. Excellent article qui analyse la polarisation de la vie et de la mort dans l'œuvre.

MAYES, Hubert-G., « Rythmes et structures dans le roman québécois de 1950 à 1965 ", thèse de doctorat, Université Laval, 1975, pp. 163-167. Brève analyse du rythme dans Les Chambres de bois qui proviendrait de la disposition des trois parties du roman. 
MERLER, Grazia, "La Réalité dans la prose d'Anne Hébert ", Ecrits du Canada français, no 33 (1971) pp. 45-83. Cet essai propose d'analyser la prose hébertienne en fonction de la technique romanesque. Vise en particulier le degré d’éloignement du lecteur et les niveaux de conscience dans Kamouraska. Outre quelques commentaires pertinents, ce travail reste en deçà d'une analyse approfondie de la technique.

MEZEI, Kathy, «Anne Hébert, Les Chambres de bois: A Translation and Interpretation ", thèse de maîtrise, Université Carleton, 1971. Très bonne traduction du roman. Discussion intelligente des thèmes et des dimensions spatio-temporelles. Étude trop rapide des élements formels.

MEZEI, Kathy, "Anne Hébert: A Pattern Repeated", Canadian Literature, No 72 (spring 1977) pp. 29-40. Cet article reprend certaines parties de la thèse cidessus mentionnée, notamment l'étude du thème de l'incapacité de vivre.

MOUSSALI, Mireille, "L'Oeuvre romanesque d'Anne Hébert", thèse de maîtrise, Université Laval, 1967. Description systématique des grands thèmes. Mais analyse peu appronfondie des techniques de l'auteur.

NADEAU-FOURNELLE, Jeanine, "Analyse des techniques narratives dans Kamouraska", thèse de maîtrise, Université du Québec à Montréal, 1977. Non vidi.

NAHMIASH, Robert, "L'Oppression et la violence dans l'œuvre d'Anne Hébert ", thèse de maitrise, Université McGill, 1972. Inventaire analytique des différentes formes d'oppression dans l'œuvre. Conclusion peu convaincante qui se rapporte aux intentions de la romancière.

NORTHEY, Margot, "Psychological Gothic: Kamouraska» dans The Gothic and Grotesque in Canadian Fiction, University of Toronto Press, 1977, pp. 53-62. Analyse originale qui met en évidence les éléments gothiques dans Kamouraska. Démontre que sur le plan du premier récit sont présents les lieux communs de la tradition gothique, notamment la violence, le meurtre, l'évasion, l'emprisonnement, la sadisme, la sexualité et surtout une atmosphère d'effroi et de suspense. Quant au deuxième récit, c'est par l'entremise des fantasmes, du dédoublement et de la destruction qu'une terreur gothique s'empare de la psyché de l'héroïne.

OUELLETTE, Gabriel-Pierre, "Espace et délire dans Kamouraska d'Anne Hébert", Voix et images, I no 2 (décembre 1975) pp. 241-264. Une discussion intelligente et détaillée du roman qui démontre que sur l'espace restreint de la chambre où Elisabeth se repose se greffent progressivement d'autres lieux qui entraînent l'héroïne dans un délire.

PAGE, Pierre, Anne Hébert, Montréal, Fides, coll. «Ecrivains canadiens d'aujourd'hui", 1965. Première introduction à l'œuvre d'Anne Hébert: vie, commentaire critique et choix de textes. Au sujet des Chambres de bois, l'auteur propose une interprétation d'ordre métaphysique du thème du refus d'être.

PARADIS, Suzanne, Fermme fictive, fermme réello, Québec, Éditions Garneau, pp. 137-143. Commentaires sur les personnages de Catherine et de Lia dans Les Chambres de bois.

POULIN, Gabrielle, "Qui sont Les Enfants du sabbat ?", Les Lettres québécoises, I no 1 (mars 1976) pp. 4-6. Bref aperçu du roman. S'interroge sur le sens métaphorique du titre.

ROBERT, Guy, La Poétique du songe: introduction à l'ceuvre d'Anne Hébert, Association générale des étudiants de l'Université de Montréal, Cahier no 4, 1962. 
Essai qui indique, à l'aide de nombreuses citations, les grands thèmes dans l'œuvre.

ROBIDOUX, Réjean et André RENAUD, "Les Chambres de bois" dans Le Roman canadien-français du vingtième siècle, Ottawa, Eds de I'Université d'Ottawa, 1966, pp. 171-185. Première étude significative des Chambres de bois. Analyse formelle qui étudie certains éléments de la composition, notamment la narration et les structures temporelles. Examine le rapport entre le roman et la poésie sur le plan de la durée.

ROBIDOUX, Réjean, "Kamouraska de Anne Hébert", Livres et auteurs québécois 1970, pp. 24-26. Excellente petite étude qui souligne la création envoûtante du roman.

SAINTE-MARIE ELEUTHERE, Sœur, La Mère dans le roman canadien-français contemporain, Québec, Les Presses Universitaires de Laval, 1964, pp. 121-126. Etude très contestable des Chambres de bois. Perçoit les chambres et l'anneau comme des symboles de la mère.

SYLVESTRE, Roger, "Du sang sur les mains blanches" Critères, no 4 (juin 1971) pp. 46-61. Cet essai se rapporte surtout à Kamouraska. Donne un résumé du roman et signale l'aspect dramatique de la structure et de l'écriture qui met en lumière le theme de la rage de vivre.

TELLES, Mercédès, “Lecture d'Anne Hébert; Kamouraska », thèse de maîtrise, Université McGill, 1971. Analyse peu approfondie de la structure, des themes et de la signification d'ensemble.

THERIO, Adrien, «La Maison de la belle et du prince ou l'enfer dans l'œuvre romanesque d'Anne Hébert", Livres et auteurs québécois 1971, pp. 274-285. Etude originale de la prose, souligne le thème central de la maison d'où découlerait celui de la belle au bois dormant.

TOMLINSON, Muriel D., "A Comparison of Les Enfants terribles and Les Chambres de bois", Revue de lUniversité d'Ottawa, 43 no 4 (octobre-décembre 1973) pp. 532-539. Commentaires sans doute justes mais superficiels.

VERONNEAU, Pierre, “Kamouraska: étude du roman et de son adaptation cinématographique", thèse de maitrise, Université du Québec à Montréal, 1977. Non vidi.

WALKER, Cheryl Anne, "Anne Hébert; The Mystery of Innocence and Experience", thèse de maîtrise, University of Western Ontario, 1961. Étude peu approfondie, à orientation biographique, du thème de la libération dans l'œuvre.

ZEGERS, John E., "La lutte entre l'esclavage et la liberté: leitmotiv dans la prose d'Anne Hébert ", North American French Language and Literature (French VIII Modern Language Association Bulletin) no 12 (décembre 1968) pp. 26-35. Non vidi.

Note: Nous tenons à remercier Christina $\mathrm{H}$. Roberts-van Oordt pour les indications bibliographiques qu'elle a eu l'amabilité de nous fournir.

Juin 1978

Janet M. PATERSON

Université de Toronto 